\title{
Modélisation de l'amplification d'une impulsion laser UV nanoseconde dans un milieu à excimères
}

\author{
O. Utéza, M. Boyomo-Onana1, Ph. Delaporte, N. Destouches, B. Fontaine, \\ M. Sabonnadière et M.L. Sentis
}

Laboratoire Lasers, Plasmas et Procédés Photoniques (LP3), FRE 2165 du CNRS, Université d'Aix Marseille II, Case 917, 163 avenue de Luminy, 13288 Marseille cedex 9, France

${ }^{1}$ École Nationale Supérieure Polytechnique, BP. 8390, Yaounde, Cameroun

Résumé. Cet article présente un code numérique temporel décrivant le transport d'un champ de rayonnement UV dans un milieu à excimères amplificateur $\mathrm{XeCl}$ et dans l'espace libre. Le modèle est ensuite appliqué à l'analyse de l'amplification d'un pulse court $\mathrm{XeCl}(\sim 3 \mathrm{~ns})$ dans un milieu actif de volume 0,1 litre.

\section{DESCRIPTION DU CODE NUMERIQUE}

La structure du code (voir fig. 1) consiste en la répétition de la séquence suivante : amplification du champ de rayonnement I dans le milieu actif puis propagation dans l'espace libre avant son retour dans le milieu amplificateur. Elle est ainsi bien adaptée à la description de systèmes oscillateur - amplificateur. Le code prend en compte l'aspect dynamique des processus d'amplification du rayonnement dans le milieu actif grâce à un découpage en tranches des impulsions et du milieu actif, la formation d'Emission Spontanée Amplifiée (ESA) dans l'amplificateur (traitée comme une source externe de bruit de photons, [1]) ainsi que les caractéristiques géométriques du système développé (disposition des miroirs, longueur des bras,...).

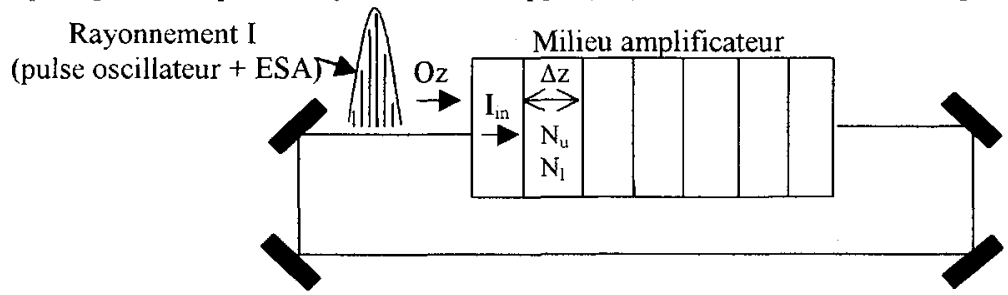

Figure 1. Structure du code ( $\mathrm{N}_{u}, \mathrm{~N}_{!}$: respectivement populations du niveau haut $\mathrm{XeCl}(\mathrm{B})$ et bas $\mathrm{XeCl}(\mathrm{X})$ de la transition laser).

Le milieu amplificateur est schématisé par un système laser à deux niveaux (transition électronique $\mathrm{XeCl}(\mathrm{B}) \rightarrow \mathrm{XeCl}(\mathrm{X})$ ) homogène dans le temps et l'espace (indice de réfraction : $\mathrm{n}=1$ ). Les équations décrivant l'amplification d'une tranche de rayonnement $\mathrm{I}_{\text {in }}$ dans une tranche de milieu actif $\Delta \mathrm{z}$ sont

$$
\begin{gathered}
\frac{d N_{u}}{d t}=R_{u}-\frac{N_{u}}{\tau_{u}}+\frac{N_{l} I_{i n} \sigma}{h v}-\frac{N_{u} I_{i n} \sigma}{h v} \\
\frac{d N_{l}}{d t}=-\frac{N_{l}}{\tau_{l}}+\frac{N_{u}}{\tau_{u}}-\frac{N_{l} I_{i n} \sigma}{h v}+\frac{N_{u} I_{i n} \sigma}{h v} \\
I_{\text {out }}=I_{\text {in }} \exp \left\{\left(g_{0, \text { eff }}-\alpha\right) \Delta z\right\}=I_{\text {in }} \exp \left\{\left(\sigma\left(N_{u}-N_{l}\right)-\alpha\right) \Delta z\right\}
\end{gathered}
$$

Les données cinétiques du milieu amplificateur (pompage $R_{u}$ et durée de vie du niveau haut $\tau_{u}$ et bas $\tau_{1}$ de la transition laser, absorption $\alpha$, section efficace d'émission stimulée $\sigma$ ) sont déterminées à partir de mesures expérimentales ou déduites de résultats admis dans la littérature des lasers à excimères. 


\section{2: APPLICATION A UN SYSTEME XECL OSCILLATEUR - AMPLIFICATEUR A 4 PASSAGES}

La figure 2 présente le système développé et précise les paramètres d'initialisation du code [2].

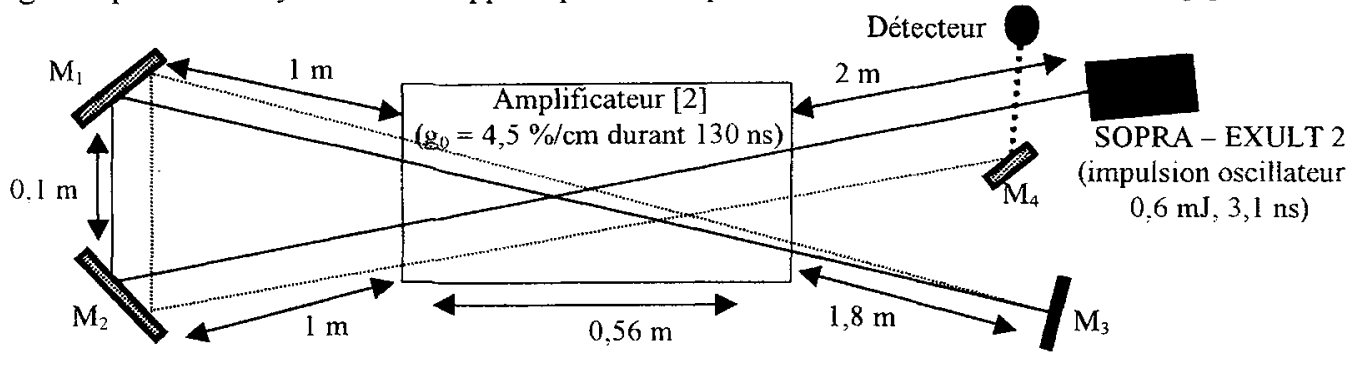

Figure 2. Système oscillateur - amplificateur à 4 passages. $M_{1}, M_{2}, M_{4}: R_{\text {max }} \dot{d} \sim 45^{\circ} . M_{3}: R_{\operatorname{tnax}} \dot{a} \sim 0^{\circ}$. Angle « off - axis 》 $2,5^{\circ} . \Delta z=25 \mu \mathrm{m}, R_{u}=3,510^{22}$ molécules. $\mathrm{s}^{-1} . \mathrm{cm}^{-3}, \tau_{\mathrm{u}}=4,9 \mathrm{~ns}, \tau_{\mathrm{i}}=0,2 \mathrm{~ns},\left(x=0,003 \mathrm{~cm}^{-1}, \sigma=2,610^{-16} \mathrm{~cm}^{-2}\right.$.

Les résultats sont décrits sur les figures $3 \mathrm{a}, \mathrm{b}$. Les paramètres $\left(1-c_{\text {seed }}\right)$ et $\left(1-c_{E S A}\right)$ représentent respectivement les pertes subies par le pulse oscillateur et l'ESA entre deux passages consécutifs dans le milieu actif.

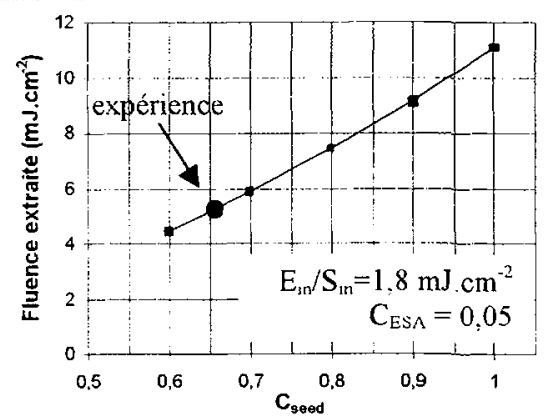

Figure 3a. Fluence extraite en fonction de $c_{\text {seecl. }}$

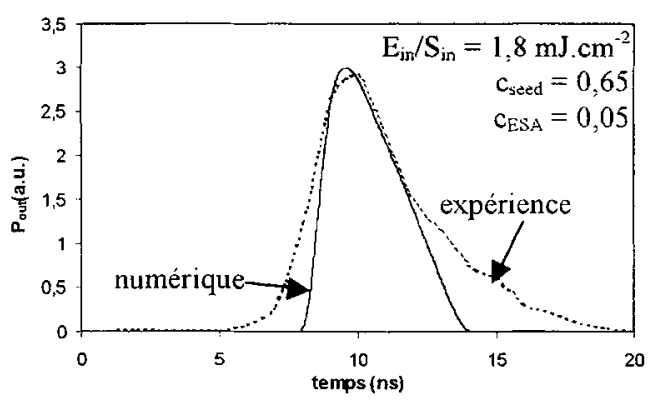

Figure 3b. Puissance crête extraite $\mathrm{P}_{\text {uut }}$ (calcul et expérience)

Les résultats expérimentaux d'amplification sont : $E_{\text {out }}=10,2 \pm 0,5 \mathrm{~mJ}, E_{\text {oul }} / S_{\text {out }}=5,1 \mathrm{~mJ} \mathrm{~cm}$, $\tau_{\text {FWHM out }}=3,8 \mathrm{~ns}$ et $\mathrm{P}_{\text {out }} \approx 2,7 \mathrm{MW}$ (soit une multiplication par 2 de la puissance crête délivrée par l'amplificateur lorsqu'il fonctionne en oscillateur [2]). Le calcul du contraste $\left(\mathrm{I}_{\text {pulse }} / \mathrm{I}_{\mathrm{ASE}}\right)$ donne $\mathrm{C}=310^{4}$ $\mathrm{l}$ (voir aussi figure $3 \mathrm{~b}$ pour une confirmation qualitative par l'expérience). Cette bonne valeur du contraste est due à un faible produit $\mathrm{g}_{0} \mathrm{l}_{\text {gain }}\left(\mathrm{g}_{0} \approx 4,5 \% \mathrm{~cm}^{-1}\right.$ et $\left.\mathrm{l}_{\text {gain }}=25 \mathrm{~cm}\right)$ et à une valeur élevée de l'angle "off axis" $\left(\sim 2,5^{\circ}\right)$ assurant une minimisation efficace de l'ESA dans l'amplificateur. L'accroissement de la largeur à mi - hauteur de l'impulsion oscillateur amplifiée $\left(\tau_{\mathrm{FWHM} \text {,out }}=3,8 \mathrm{~ns}\right.$; calcul: $\tau_{\mathrm{FWHM} \text {.out }}=3,3 \mathrm{~ns}$ et $\tau_{\text {FWHM.in }}=3,1 \mathrm{~ns}$ ) est dû à une amplification significative des bords temporels de l'impulsion. L'efficacité énergétique des processus d'amplification est en effet maximum pour $\left.\mathrm{E}_{\mathrm{in}} \approx \mathrm{E}_{\mathrm{sat}}[3]\left(\mathrm{E}_{\mathrm{sat}} \approx 2,5 \mathrm{~m}\right] \mathrm{cm}^{-2}[1]\right)$.

Enfin, l'accord entre les résultats expérimentaux et numériques est obtenu pour $\mathrm{c}_{\text {seed }}=0,65$ (soit $35 \%$ de pertes pour le pulse oscillateur entre 2 passages). Les pertes importantes subies par l'impulsion oscillateur sont principalement dues à la présence d'éléments optiques de dimensions utiles faibles $\left(\varnothing_{\text {lenitre.ampli. }}=4,2 \mathrm{~cm}\right.$, hauteur inter - électrodes $\left.=3 \mathrm{~cm}, \ldots\right)$ qui diaphragment rapidement le faisceau. L'amélioration de la géométrie de la cavité (réduction de sa longueur,...) et un meilleur choix des éléments optiques (optiques de plus grand diamètre, recours à des miroirs sphériques, ...) devraient ainsi permettre d'accroître les performances énergétiques de ce système oscillateur - amplificateur.

\section{Références}

[1] P.B. Corkum, R.S. Taylor, IEEE J. of Quantum Electron., 18 (11), (1982) 1962.

[2] O. Utéza, M. Boyomo - Onana, Ph. Delaporte, N. Destouches, B. Fontaine, M. Sabonnadière, M Sentis, soumise à Optics Comm.

[3] G. Almasi, S. Szatmari, P. Simon, Optics Comm. 88 (1992) 231. 